\title{
Frictional behavior of nanostructured carbon films
}

\author{
Dongfeng DIAO ${ }^{1,2, *}$, Chao WANG ${ }^{2}$, Xue FAN ${ }^{1, *}$ \\ ${ }^{I}$ Key Laboratory of Education Ministry for Modern Design and Rotor-Bearing System, School of Mechanical Engineering, Xi'an Jiaotong \\ University, Xi'an 710049, China \\ ${ }^{2}$ Nanosurface Science and Engineering Research Institute, Shenzhen University, Shenzhen 518060, China \\ Received: 02 November 2012 / Revised: 16 January 2013 / Accepted: 01 February 2013 \\ (C) The author(s) 2013. This article is published with open access at Springerlink.com
}

\begin{abstract}
We propose a new path for preparing nanostructured carbon films (NCFs) by using electron cyclotron resonance (ECR) plasma sputtering with ion-electron hybrid irradiation for controlling the frictional behavior. The frictional behavior of the NCF was measured by using a pin-on-disk tribometer with a nanoprobe displacement sensor, and the transition curves of the friction coefficient and microdisplacement of the NCFs were examined. The friction mechanism was discussed by transmission electron microscopy (TEM) observation on the wear track. From the results, we found a new method to prepare NCFs, which has the potential to achieve low friction at the early stage of sliding contact. In addition, the technology of ECR plasma with ion-electron hybrid irradiation provides a new vision to rebuild a nanostructured surface from an original surface for controlling the frictional behavior.
\end{abstract}

Keywords: friction; nanostructured carbon film (NCF); electron cyclotron resonance (ECR); ion-electron hybrid irradiation

\section{Introduction}

Amorphous carbon films exhibit low friction coefficient and long wear life, which makes them suitable for tribological applications as they provide protection for counterparts. To date, many studies on the friction and wear mechanisms of amorphous carbon films have been published, and the friction and wear behaviors were affected by the intrinsically chemical, structural, and mechanical properties of the films, as well as the externally chemical, physical, and mechanical interactions between the amorphous carbon film surfaces and their surroundings [1-10]. Recently, nanostructured carbon films (NCFs) with graphene-like nanocrystallites have attracted a lot of interest because they not only show good tribological performance but also high electrical conductivity [11, 12]. Such materials have many potential applications as novel coating materials in the nanotribology field.

\footnotetext{
* Corresponding author: Xue FAN, Dongfeng DIAO.

E-mails: fanx@mail.xjtu.edu.cn,dfdiao@mail.xjtu.edu.cn
}

There are some researches focusing on the growth of carbon films with graphite-like nanocrystallites. Alexandro et al. [13] prepared nanocrystallite carbon film with the $\mathrm{sp}^{2}$ network structure using laser-arc evaporation and found that the film had high hardness and elastic recovery. Hirono et al. [14] reported a carbon nanocrystallite film prepared with the electron cyclotron resonance (ECR) sputtering method, and studied its electrical conductivity and hardness. Lau et al. $[15,16]$ prepared tetrahedral amorphous carbon (ta-C) film using the filtered cathodic vacuum arc (FCVA) method and studied the thermally induced $\mathrm{sp}^{2}$ clustering in the ta-C film. Orwa et al. [17] deposited ta-C film with different impact energies of carbon ions, and found that the structure of the ta- $C$ films could transform from amorphous into a $\mathrm{sp}^{2}$ rich structure containing graphite layers. Our research on the electron or ion irradiation of various carbon films demonstrated that it facilitates nanostructure formation, and that the electron or ion irradiation can be used to precisely tailor the structure and the properties of the carbon film [11, 12, 18, 19]. In this paper, we reported 
recent advances in the ECR plasma technology with particular emphasis on the ion-electron hybrid irradiation effect on the nanostructure formation in carbon films. The frictional behaviors of the films were measured by a pin-on-disk tribometer installed a nanoprobe displacement sensor for understanding the friction mechanism and the friction-induced microdisplacement of NCFs.

\section{Experiments}

\subsection{Principle of ECR plasma sputtering with ion- electron hybrid irradiation}

We previously reported [18] that in order to generate the ECR plasma, a $2.45 \mathrm{GHz}$ microwave is introduced to the chamber through a rectangular waveguide and a fused quartz window. Magnetic coils are arranged around the chamber to achieve a microwave ECR condition (magnetic flux density, $875 \mathrm{G}$ ). In the ECR plasma, when the ions transfer from a strong magnetic field to a weaker field, they are accelerated (decelerated when the ions transfer from a weak to a stronger field) by the force $F_{z}=-\mu_{z} \operatorname{gradB}_{z}$, where $\mu_{z}=m v^{2} / 2 B$ is the magnetic momentum of the plasma volume unit, and $\operatorname{gradB}_{z}$ is the gradient of the magnetic flux density $B$ in the parallel direction to the magnetic field. To understand the ion-electron hybrid irradiation process in the ECR plasma, we describe the process that is also shown in Fig. 1. Figure 1(a) is simplified to emphasize the formation of the electron irradiation and Fig. 1(b) shows the ion irradiation. Electrons are resonantly accelerated to move around the magnetic field lines, and the momentum at the magnetic mirror position (dashed lines in Fig. 1(a)) is zero along the horizontal direction. By taking this advantage and considering the floating bias voltage $\left(V_{\mathrm{f}}\right)$ at the substrate surface, the ion-electron hybrid irradiation can be realized as follows. The substrate is located near the magnetic mirror position, and when the substrate bias voltage $\left(V_{\mathrm{b}}\right)$ is higher than the $V_{\mathrm{f}}$, the electrons gain kinetic energy from $\left(V_{\mathrm{b}}-V_{\mathrm{f}}\right)$ and irradiate the film. In contrast, if $V_{\mathrm{b}}$ is lower than $V_{\mathrm{f}}$, the ions are accelerated to the substrate forming the ion irradiation. Therefore, the ion-electron hybrid irradiation can be achieved by changing substrate bias voltage higher (electron irradiation, as shown in Fig. 1(a)) and lower (ion irradiation, as shown in Fig. 1(b)) than the floating bias voltage.

\subsection{Pin-on-disk tribometer with nanoprobe displacement sensor}

The schematic illustration of the pin-on-disk tribometer with a nanoprobe displacement sensor is shown in Fig. 2. The whole image of the test rig is shown in Fig. 2(a). The design consists of a pin-on-disk tribometer and a nanoprobe displacement sensor. The pin-on-disk tribometer includes a rotating platform, a friction beam, and a three-axis $(X, Y, Z)$ manual stage (II). As shown in Fig. 2(b), a SiC ball (radius of $4 \mathrm{~mm}$ ) is fixed at the free end of the friction beam. Strain gauges are attached on the friction beam to measure the normal force and the tangential force. The specimen is fixed on the rotating platform and the position can be adjusted. The relative position between the axis of the $\mathrm{SiC}$ ball and the specimen is controlled by adjusting the three-axis manual stage (II) in the $X$ and $Y$
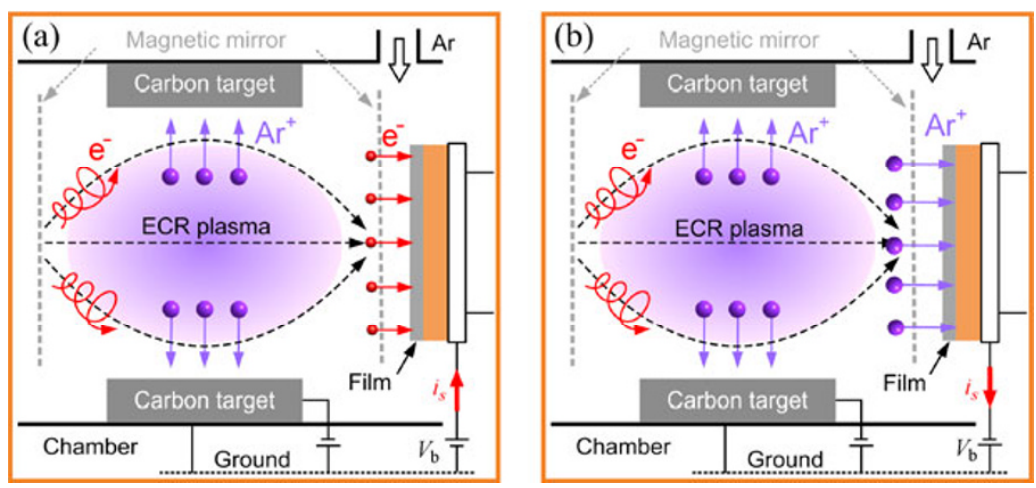

Fig. 1 Principle scheme of ECR plasma sputtering for electron irradiation (a) and ion irradiation (b). 


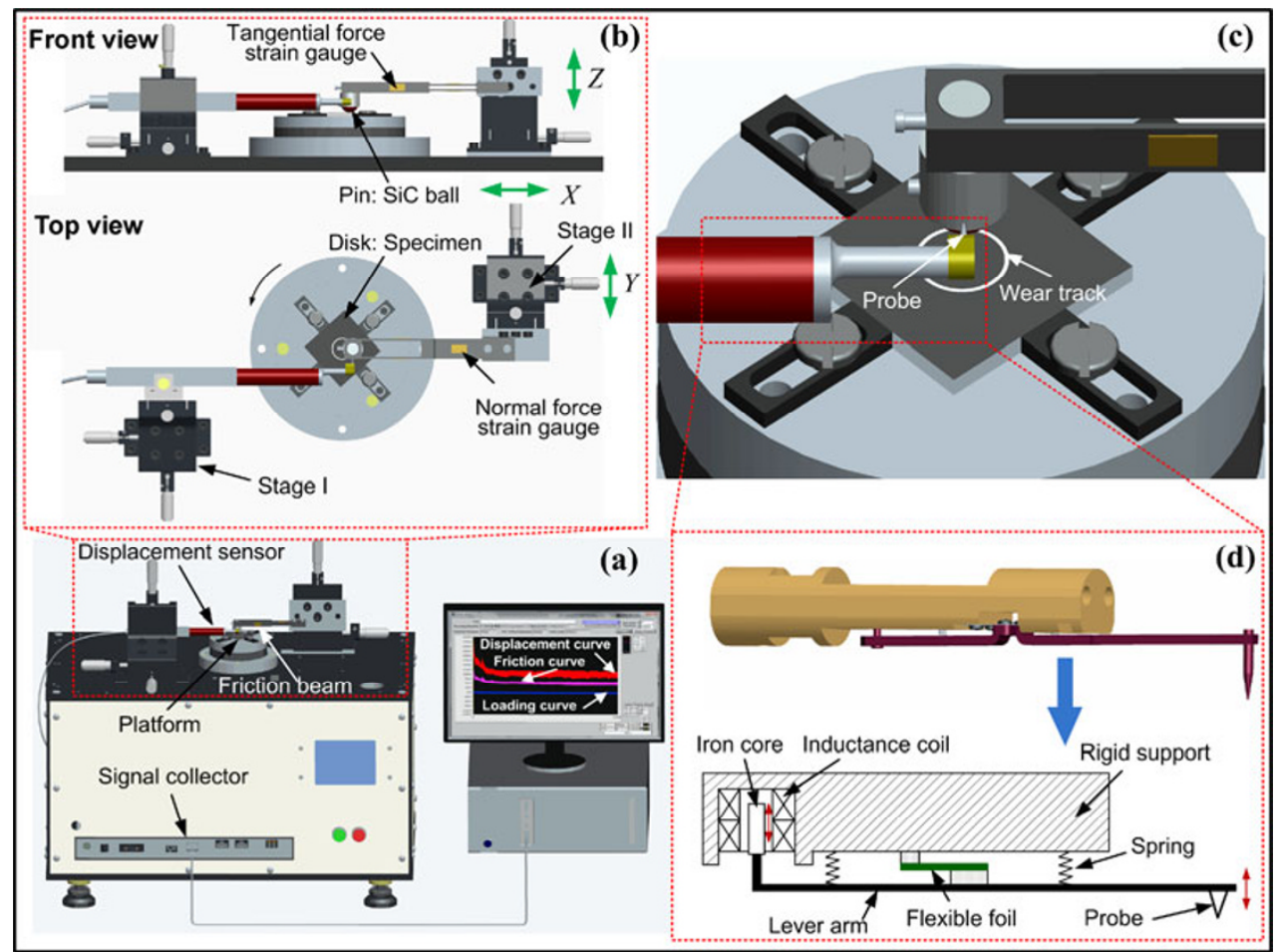

Fig. 2 Schematic illustration of pin-on-disk tribometer installed a nanoprobe displacement sensor: (a) overview of the test rig; (b) top and front views of the test parts; (c) enlarged view of the contact part of pin and sample, together with the nanoprobe; (d) working principle diagram of the nanoprobe displacement sensor.

directions, and the normal force is loaded and unloaded from specimens by adjusting the screw bolt in the $Z$ direction. The design ensures that the friction beam holds the SiC ball horizontally. The axis of the friction beam and the center of the rotating platform are in line, thus, the sliding velocity of each contact point in the tangential direction has the same value. The signals of normal load and friction force are collected and then the friction coefficient is calculated.

The nanoprobe displacement sensor consists of a displacement sensor with a nanoprobe and a threeaxis manual stage (I), as shown in Fig. 2(b). The sensor is mounted parallel to the axis of the friction beam and the probe is contacted with the $\mathrm{SiC}$ ball holder to measure the actual microdisplacement in the tangential direction during friction (see Fig. 2(c), it should be noticed that the contact force of probe for microdisplacement measurement is $0.75 \mathrm{mN}$, which is much smaller than the friction force). The measuring range of the displacement sensor is $350 \mu \mathrm{m}$ with a resolution of $0.01 \mu \mathrm{m}$. As shown in Fig. 2(d), the inductance coils are placed inside the rigid support.
The lever arm is fixed on the rigid support by using a flexible foil and the iron core is bonded at the end of the lever arm. Two springs are used to control the relative position between the lever arm and the rigid support. When the probe detects a displacement, the insert length of the iron core into the inductance coils is changed, resulting in the change of the magnetic flux. Then the voltage change is collected by the signal collector and recorded. After calibration, the microdisplacement is measured. In this study, the tribotests are performed in room temperature with a relative humidity of about $70 \%$ under a normal load $2 \mathrm{~N}$ and rotational speed of $180 \mathrm{rpm}$ with frictional radius of $1.4 \mathrm{~mm}$ (the sliding velocity is calcultatd to be $26 \mathrm{~mm} / \mathrm{s}$ ).

\subsection{Preparation and characterization of nanostructured carbon films}

Carbon films were deposited on p-type $<100>$ oriented silicon substrates with the size $20 \mathrm{~mm} \times 20 \mathrm{~mm} \times$ $0.5 \mathrm{~mm}$. The substrates were cleaned with acetone and absolute ethyl alcohol before put into the vacuum 
cavity. The background pressure of the vacuum chamber was $3 \times 10^{-4} \mathrm{~Pa}$, and argon was inflated keeping the working pressure at $4 \times 10^{-2} \mathrm{~Pa}$. The silicon substrates were pre-sputtered by argon ions to remove the residual contaminants. Then, argon ions were attracted to the carbon target to sputter the target surface, providing the carbon atoms for film growth. It was measured that floating voltage at the position of the sample located is zero when the working pressure is $0.04 \mathrm{~Pa}[11,12]$, then positive and negative DC biases of $50 \mathrm{~V}$ were applied to the substrate to realize the $50 \mathrm{eV}$ electron irradiation and the $50 \mathrm{eV}$ ion irradiation during deposition, respectively. Furthermore, to study the ion-electron hybrid irradiation, carbon film was first deposited with the $50 \mathrm{eV}$ ion irradiation and, then, the film was irradiated with $50 \mathrm{eV}$ electrons as the post-deposition processing. The current density was $63.66 \mathrm{~mA} \cdot \mathrm{cm}^{-1}$ and $1.8 \mathrm{~mA} \cdot \mathrm{cm}^{-1}$ for the electron and ion irradiation, respectively.

The nanostructures of the carbon films were observed with a JEOL 2010 transmission electron microscope (TEM). The bonding structures of the carbon films prepared with different irradiation methods were studied by using the Raman spectra between $1100 \mathrm{~cm}^{-1}$ and $3400 \mathrm{~cm}^{-1}$, which were obtained with a HORIBA HR800 laser confocal Raman spectrometer. The surface morphologies were tested with a SHIMADZU SPM9700 atomic force microscope (AFM), which worked in the dynamic observation mode with a $X, Y$ resolution of $0.2 \mathrm{~nm}$ and a $Z$ resolution of $0.01 \mathrm{~nm}$.

\section{Experimental results}

\subsection{Characterization of nanostructured carbon films}

The TEM images of the nanostructures of the carbon films are shown in Fig. 3. As shown in Fig. 3(a), when electron irradiation was used, a nanocrystalline structure with graphene sheets stacks appeared in the films. The graphene sheet stacks are uniformly distributed in the plane view and grew vertically to the substrate direction (along the cross-sectional view). Each stack contains a few graphene sheets that are linked with each other by twisting and curving. The interplanar spacing is $0.36 \mathrm{~nm}$. The electron diffraction (ED) patterns of an electron-irradiated

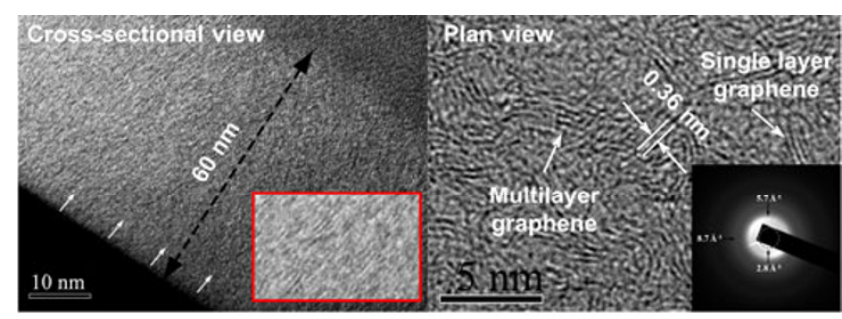

(a)

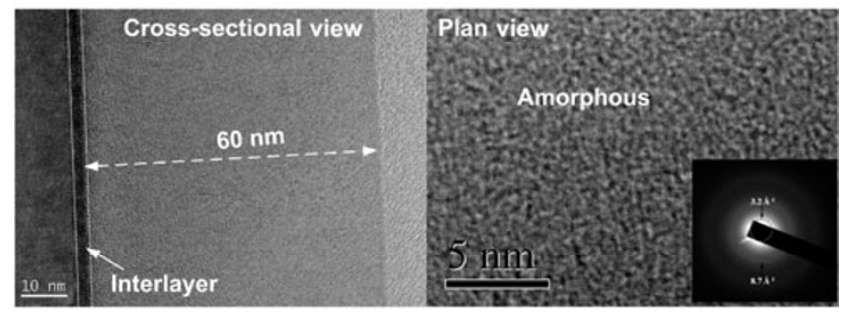

(b)

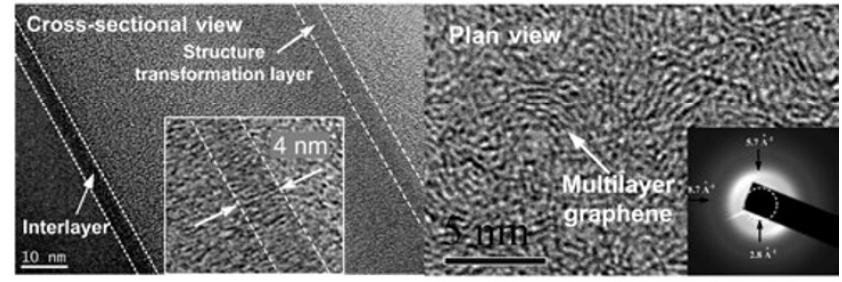

(c)

Fig. 3 TEM images of the carbon films with different irradiation methods. (a) Nanostructure of e-NCF in cross-sectional view (left) and plan view (right); (b) Nanostructure of i-CF in cross-sectional view (left) and plan view (right); (c) Nanostructure of i/e-NCF in cross-sectional view (left) and plan view (right).

nanostructured carbon films (e-NCF) showed diffraction rings, indicating a more ordered structure. The structure of an ion-irradiated carbon film (i-CF) is shown in Fig. 3(b), and is a pure amorphous structure. An ion-electron hybrid-irradiated carbon film (i/e-NCF) is shown in Fig. 3(c) in which the film structure is mainly amorphous, but the top layer with thickness of $4 \mathrm{~nm}$ was changed to graphene sheets embedded in the structure after electron irradiation.

Figure 4 shows the Raman spectra of the carbon films. The spectrum of e-NCF showed that the $D$ and $G$ bands are separate and located near $1340 \mathrm{~cm}^{-1}$ and $1600 \mathrm{~cm}^{-1}$, respectively, and the spectra showed a medium strength 2D band centered near $2700 \mathrm{~cm}^{-1}$. This proves the existence of graphene sheets in e-NCF. For the i-CF, there was only a composite band centered around $1500 \mathrm{~cm}^{-1}$, consisting of D and G bands, without $2 \mathrm{D}$ band in the Raman spectrum. In the Raman spectrum of i/e-NCF, the locations of the 


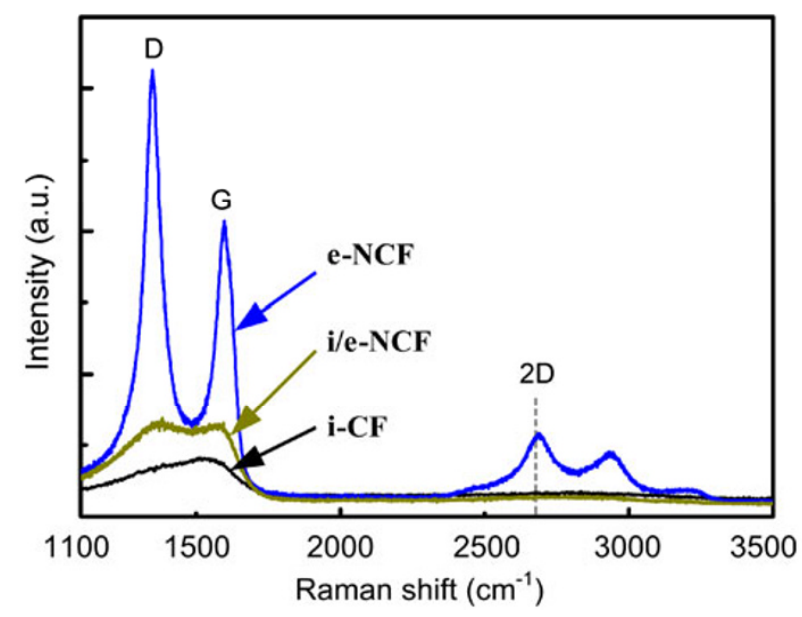

Fig. 4 Raman spectra of the carbon films with different irradiation methods.

$D$ and $G$ bands are separate but with no $2 \mathrm{D}$ band either. As studied before [11], the growth mechanism of the graphene sheets embedded in the structure was interpreted as the inelastic scattering of low energy electrons. Whereas, in the case of $\mathrm{i}-\mathrm{CF}$, the ion irradiation mechanism is the elastic and inelastic collision between ions and atoms, and weakly bonded carbon atoms are sputtered during this process. The different growth mechanisms of e-NCF and i-CF resulted in the different Raman spectra.

Figure 5 shows the surface morphologies of the carbon films produced with the different irradiation methods. From the AFM image of e-NCF (Fig. 5(a)), we see that the surface is rough with a mean surface roughness ( $\mathrm{Ra}$ ) of $13.42 \mathrm{~nm}$ and there are many prominences on the surface. When ions were used for irradiation, the surface morphologies of i-NCF get smoother with an Ra of $0.087 \mathrm{~nm}$, as shown in Fig. 5(b).
The surface image of $\mathrm{i} / \mathrm{e}-\mathrm{NCF}$, which is similar to that of $\mathrm{i}-\mathrm{CF}$, is shown in Fig. 5(c) and the mean surface roughness of i/e-NCF decreased to $0.08 \mathrm{~nm}$ when the top surface changed to graphene sheets embedded in the structure.

\subsection{Frictional behavior of nanostructured carbon films}

The pin-on-disk tribometer with a nanoprobe displacement sensor was used to investigate the frictional behavior. The transition curves of the friction coefficient together with the microdisplacement of the carbon films are shown in Fig. 6. The inserted graphs show the oscillation amplitude of the microdisplacement. The friction coefficient of the e-NCF was 0.175 at the beginning of the test and decreased to a mean value of 0.137 after about 200 cycles of cyclic friction, as shown in Fig. 6(a). Then, the friction coefficient suddenly increased after about 475 cycles, which represents the sliding of the $\mathrm{SiC}$ ball against the silicon substrate. The mean microdisplacement of the SiC ball was $107.28 \mu \mathrm{m}$ when sliding against e-NCF. For the i-CF, the friction coefficient gradually decreased from 0.180 and stabilized at a mean value of 0.075 at 1000 frictional cycles, and the mean microdisplacement of SiC ball was $37.28 \mu \mathrm{m}$, as shown in Fig. 6(b). The friction coefficient transition curve of $\mathrm{i} / \mathrm{e}-\mathrm{NCF}$ is shown in Fig. 6(c), and is similar to that of i-CF with a stabilized mean friction coefficient of 0.079 and a mean microdisplacement of $46.20 \mu \mathrm{m}$. However, the sliding cycles for reaching low friction coefficient is only 200 cycles, which is much quicker than the $\mathrm{i}-\mathrm{CF}$.
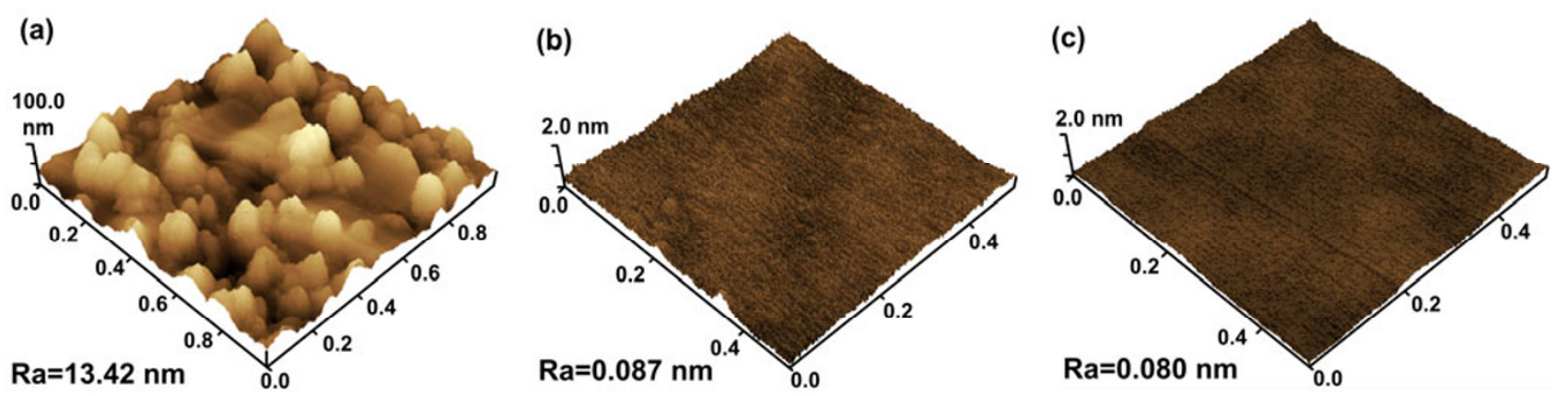

Fig. 5 Surface morphologies of the carbon films: (a) e-NCF, (b) i-CF, and (c) i/e-NCF. 

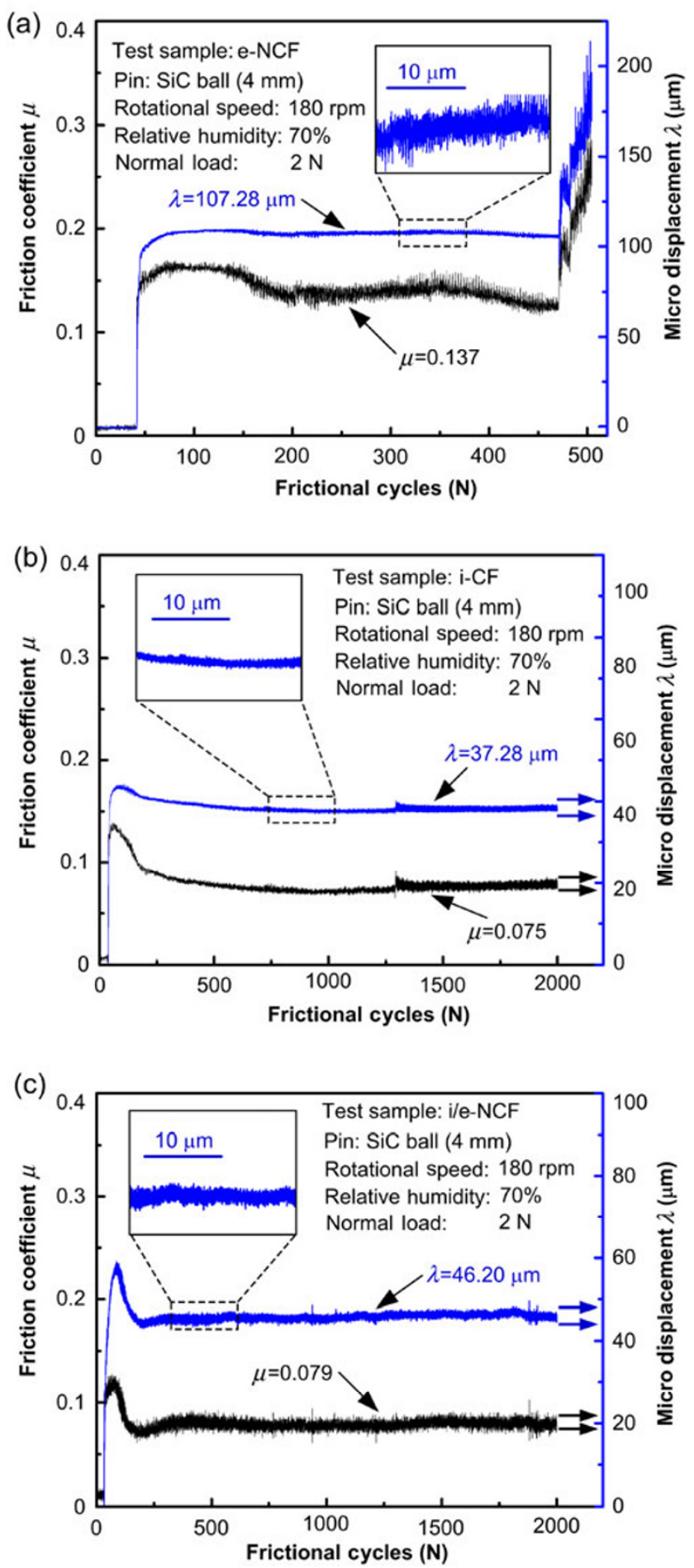

Fig. 6 The transition curves of friction coefficient and microdisplacement of the carbon films: (a) e-NCF, (b) i-CF, and (c) $\mathrm{i} / \mathrm{e}-\mathrm{NCF}$.

To understand the frictional behavior of nanostructured carbon films, Fig. 7 shows the TEM images of the low frictional wear tracks of the e-NCF and i-CF, respectively. In Fig. 7(a), the wear
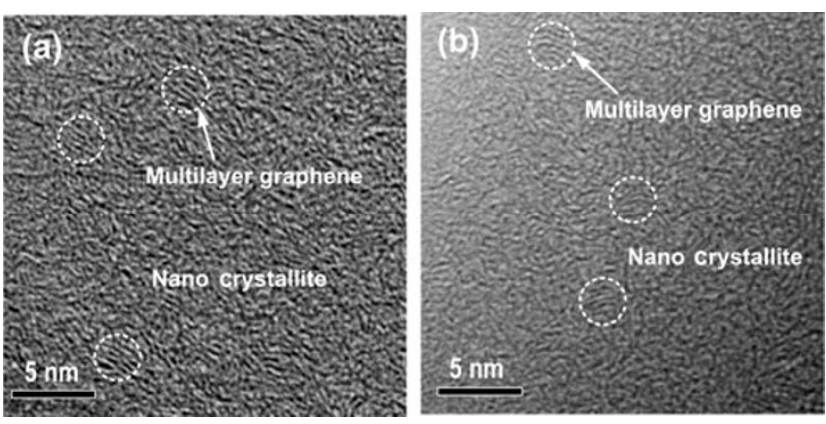

Fig. 7 TEM images of low frictional wear tracks of (a) e-NCF, and (b) i-CF.

track of e-NCF slightly changed into a less organized nanostructure than the originally deposited surface, it remains nanocrystalline structure. Comparing Fig. 7(b) with Fig. 3(b), it is clear that the amorphous structure of i-CF changed into a nanocrystalline structure containing graphene sheets stacks.

Concerning the low friction behavior of amorphous carbon films, there are two widely accepted explanations which are the formation of a transfer film on the counter sliding surface and the graphitization of the transfer film, and those were studied in our earlier research [12, 19]. The TEM image of the wear track provided direct evidence of the structural transformation from amorphous to graphene sheets embedded in the structure during cyclic friction, which we call "graphenization". The graphenization process can be one of the reasons why the friction coefficient decreased.

By comparing the TEM images of the e-NCF, i-CF, and their wear tracks, we found that the wear track of amorphous carbon film in the low friction stage has the similar structure with the e-NCF structure. From the tribotest result of $\mathrm{i}-\mathrm{CF}$, it can be seen that before the low friction stage, there is a high friction stage and a gradually-decreasing stage that took about 1000 frictional cycles, as shown in Fig. 6(b). This stage is not preferred when we consider the carbon films as a lubricant coating. Then it is necessary to shorten or even avoid the high friction stage. Since the nanostructure in e-NCF is similar with the low friction stage carbon, it may make the film reach low friction at early stage. In order to achieve low friction at the beginning of the sliding, the ion-electron hybrid irradiated film was prepared. As shown in Fig. 3(c), the basic structure of the i/e-NCF obtained by 
ion irradiation is amorphous, whereas the top layer nanostructure was modified from amorphous to graphene sheets embedded in the structure by electron irradiation. Figure 6(c) shows that the critical cycles for achieving a low friction coefficient is 200 cycles and the wear life is longer than 2000 cycles with a low friction coefficient of 0.079 .

\section{Discussion}

The frictional behaviors of nanostructured carbon films were obtained by using a pin-on-disk tribometer with a nanoprobe displacement sensor. It should be noted that the stabilized low friction coefficient of e-NCF (0.137) is nearly twice higher than that of i-CF (0.075) and i/e-NCF (0.079). Concerning the mechanism, we consider that the original surface roughness may play an important role. It can be seen in Fig. 5(a) that the roughness of e-NCF is $13.42 \mathrm{~nm}$, whereas in the cases of i-CF and i/e-NCF, the value is $0.087 \mathrm{~nm}$ and $0.080 \mathrm{~nm}$, respectively. The big difference in surface roughness may affect the friction coefficient of the nanostructured carbon films, the values of the low friction coefficient of i-CF and i/e-NCF with similar surface roughness are nearly the same, and the "graphenization" of NCFs during friction affects the friction coefficient decreasing at the early stage.

On the other hand, the frictional transition curves also showed that the wear life of e-NCF (475 cycles) is shorter than that of $\mathrm{i}-\mathrm{CF}$ and $\mathrm{i} / \mathrm{e}-\mathrm{NCF}$ ( $>2000$ cycles). The wear life of carbon films depends on the interface strength. In the e-NCFs, during the film growth, the electrons exchange energy with the valence electrons of the carbon atoms through inelastic scattering to modify the bonding states and they cannot change the atom position during deposition. The film growth rate along the thickness direction (vertical growth) is quicker than that along the horizontal direction, and the graphene sheets structure may grow freely in one direction with low bonding energy. As a result, the surface morphology in Fig. 5(a) shows prominences, and the graphene sheets stacks randomly distributed in the plan view (see Fig. 3(a)), resulting in a much weaker interface strength between the e-NCF and the substrate due to their lattice-mismatch. In contrast, when the films are deposited under ion irradiation, the weak bonds between carbon atoms are broken by the energetic argon ions and then the carbon atoms can spread in the horizontal direction, leading to a much stronger bonding state and a smoother surface (see Fig. 5(b)). Furthermore, in the case of i-CF, the interdiffusion takes place at the initial stage of film growth under ion irradiation, which may form an interlayer containing $\mathrm{Si}-\mathrm{Si}, \mathrm{C}-\mathrm{Si}$, and $\mathrm{C}-\mathrm{C}$ bonds. The interlayer plays an important role in increasing the bonding strength between the film and the substrate $[18,20-21]$. In i/e-NCF, only the nanostructure of the top layer was modified, the structure near the interface remains the i-CF structure, which results in a stronger interfacial strength. Therefore, i-CF and i/e-NCF with stronger bonding interface exhibited longer wear life. The interface bonding strength of e-NCF still needs to be improved for further study.

As discussed above, in order to control the frictional behavior of NCFs, the merits of smooth surface for low friction coefficient, high interface strength for long wear life, and graphene sheets embedded in the nanostructure for achieving low friction coefficient quickly were integrated with the ion-electron hybrid irradiation method. The i/e-NCFs have not only excellent frictional properties, but also good electric conductivity [11] and magnetic behavior [22]. As a result, the i/e-NCFs can find wide use in applications with outstanding tribological, electrical and megnetical properties.

Finally, in this paper, the nanoprobe displacement sensor was installed on a pin-on-disk tribometer, which is used to in-situ monitor the microdisplacement of the SiC ball when sliding against the carbon films for understanding the effect of friction-induced microdisplacement on the friction mechanism. By using the nanoprobe displacement sensor, except that the microdisplacement of the $\mathrm{SiC}$ ball during friction is obtained, and the oscillation amplitude of the microdisplacement also can be quantified (as shown by the small inserted blocks in Fig. 6), which represents the friction induced oscillation of the pin. For example, the oscillation amplitude of e-NCF is larger than those of $\mathrm{i}-\mathrm{CF}$ and $\mathrm{i} / \mathrm{e}-\mathrm{NCF}$, and this phenomenon may be caused by the surface roughness. 
Thus, the surface roughness of e-NCF is higher than that of $\mathrm{i}-\mathrm{CF}$ and $\mathrm{i} / \mathrm{e}-\mathrm{NCF}$, and the contact, the friction, the mechanical interlocking, and the peeling of large asperities will generate larger amplitude friction oscillation. When the NCFs are used in the precision instruments, the friction-induced microdisplacement and its oscillation amplitude at the contact interface play an important role for effecting precise positioning, therefore, in-situ tracking the friction vibration is necessary.

\section{Conclusions}

A new path for preparing NCFs for controlling friction coefficient was proposed by using the method of ECR plasma sputtering combined with ion-electron hybrid irradiation manufacturing. The pin-on-disk tribometer with a nanoprobe displacement sensor for measuring the frictional behavior of the film was developed for better understanding of the friction mechanism of the NCFs. The transition curves of the friction coefficient and the microdisplacement of the NCFs were summarized, and the TEM observations on the low frictional wear track indicated that the NCFs have the potential to achieve low friction coefficient at the early stage of cyclic friction. These findings help to understand the frictional behavior of nanostructured carbon films.

\section{Acknowledgements}

The authors want to thank the National Nature Science Foundation of China under Nos. of 90923027 and 51175405.

Open Access: This article is distributed under the terms of the Creative Commons Attribution Noncommercial License which permits any noncommercial use, distribution, and reproduction in any medium, provided the original author(s) and source are credited.

\section{References}

[1] Robertson J. Diamond like amorphous carbon. Mater Sci Eng 37: 129-132 (2002)

[2] Lu W, Komvopoulos K. Nanotribological and nanomechanical properties of ultrathin amorphous carbon films synthesized by radio frequency sputtering. ASME J Tribo 123: 641-650 (2001)

[3] Erdemir A, Donnet C. Tribology of diamond-like carbon films: Recent progress and future prospects. J Phys D: Appl Phys 39: R311-R327 (2006)

[4] Dickrell P L, Argibay N, Erylimaz O L, Erdemir A, Sawyer W G. Temperature and water vapor pressure effects on the friction coefficient of hydrogenated diamond like carbon films. ASME J Tribo 131: 032102 (2009)

[5] Eryilmaz O L, Erdemir A. TOF-SIMS and XPS characterization of diamond-like carbon films after tests in inert and oxidizing environments. Wear 265: 244-254 (2008)

[6] Heimberg J A, Wahl K J, Singer I L, Erdemir A. Superlow friction behavior of diamond-like carbon coatings: Time and speed effects. Appl Phys Lett 78: 2449-2451 (2001)

[7] Sugimoto I, Miyake S. Oriented hydrocarbons transferred from a high performance lubricative amorphous C:H:Si film during sliding in a vacuum. Appl Phys Lett 56: 1868-1870 (1990)

[8] Fukui H, Irie M, Utsumi, Y, Oda K, Ohara, H. An investigation of the wear track on DLC (a-C:H) film by time-of-flight secondary ion mass spectroscopy. Surf Coat Technol 146: 378-383 (2001)

[9] Holmberg K, Ronkainen, H, Laukkanen, A. Friction and wear of coated surfaces-scales, modelling and simulation of tribomechanisms. Surf Coat Technol 202: 1034-1049 (2007)

[10] Scharf T W, Singer I L. Role of transfer film on the friction and wear of metal carbide reinforced amorphous carbon coatings during run-in. Tribo Lett 36: 43-53 (2009)

[11] Wang C, Diao D F, Fan X, Chen C. Graphene sheets embedded carbon film prepared by electron irradiation in ECR plasma. Appl Phys Lett 100: 231909 (2012)

[12] Wang C, Diao D F. Cross-linked graphene layer embedded carbon film prepared using electron irradiation in ECR plasma sputtering. Surf Coat Technol 206: 1899-1904 (2011)

[13] Alexandrou I, Scheibe H J, Kiely C J, Papworth A J, Amaratunga G A J, Schultrich B. Carbon films with an $\mathrm{sp}(2)$ network structure. Phys Rev B 60: 10903-10907 (1999)

[14] Hirono S, Umemura S, Tomita M, Kaneko P. Superhard conductive carbon nano crystallite films. Appl Phys Lett 80: 425-427 (2002)

[15] Lau D W M, McCulloch D G, Taylor M B, Patridge J G, Mckenzie D R, Marks N A, Teo E H T, Tay B K. Abrupt stress induced transformation in amorphous carbon films with a highly conductive transition phase. Phys Rev Lett 100: 17601 (2008) 
[16] Lau D W M, Moaf A, Taylor M B, Partridge J G, McCulloch D G, Powles R C, McKenzie D R. The structural phases of non-crystalline carbon prepared by physical vapor deposition. Carbon 47: 3263-3270 (2009)

[17] Orwa J O, Andrienko I, Peng J L, Prawer S, Zhang Y B, Lau S P. Thermally induced $\mathrm{sp}^{2}$ clustering in tetrahedral amorphous carbon (ta-C) films. J Appl Phys 96: 6286-6297 (2004)

[18] Fan X, Diao D F, Wang K, Wang C. Multi-functional ECR plasma sputtering system for preparing amorphous carbon and Al-O-Si films. Surf Coat Technol 206: 1963-1970 (2011)

[19] Fan X, Diao D F. Contact mechanisms of transfer layered

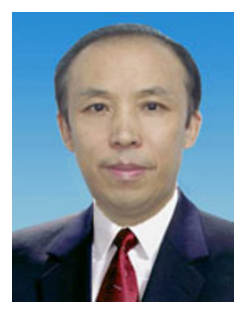

Dongfeng DIAO. Professor, obtained his PhD degree in 1992 from Tohoku University, Japan. He worked as a research associate from 1993 to 1994 in Tohoku University, and as an associate professor from 1995 to 2002 in Shizuoka University, Japan. During the period, he was invited to the Ohio State University from March to August, 1996 as a JSPS Research Fellow. He was appointed as a "Tengfei" special professor in 2002, and a full professor in 2005 in Xi'an Jiaotong University, China. He was appointed as the director of Laboratory of Education Ministry for Modern Design and Rotor-Bearing System in 2003 in Xi'an

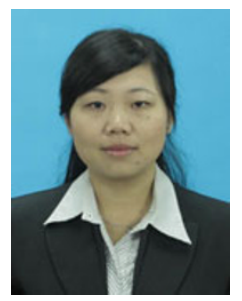

Xue FAN. Assistant professor, obtained her Bachelor degree in 2006 and PhD degree in 2012 from Xi'an Jiaotong University. She worked with Prof. Toyonobu Yoshida in the University of Tokyo from October 2010 to November 2011, and now she is working with Prof. Dongfeng Diao as a post doctor in Xi'an Jiaotong University. She worked as an assistant professor since 2012 at Key Laboratory of surface during sliding wear of amorphous carbon film with finite element analysis. ASME J Tribo133: 042301 (2011)

[20] Kelires P C, Gioti M, Logothetidis T S. Interfacial stability and atomistic processes in the a-C/Si.100 heterostructure system. Phys Rev B 59: 5074-5081 (1999)

[21] Patsalas P, Logothetidis T S, Kelires P C. Surface and interface morphology and structure of amorphous carbon thin and multilayer films. Diamond Relat Mater 14: 1241-1254 (2005)

[22] Wang C, Diao D F. Magnetic behavior of graphene sheets embedded carbon film orginated from graphene nanocrystallite. Appl Phys Lett 102: 052402 (2013)

Jiaotong University, and as a director of Nanosurface Science and Engineering Research Institute (NSER) in 2012 in Shenzhen University. His biography has been listed in 18th edition of Who's Who Books in the world. And he is in the Editoral Board of journal of Lubrication Science and an Associated Editor of the journal of Friction. His research interest is nanosurface science and engineering with emphasis on mechanical science, FEM-MD contact mechanics, ECR plasma physics, AFM biomechanics and nanotribology. He has published more than 150 publications in various archival journals, conference proceedings, and has 10 China patents. His best paper has been cited by SCI papers for more than 110 times.

Education Ministry for Modern Design and Rotor-Bearing System. Her interested research areas include nanosurface machining, nanosurface contact mechanics and low friction and wear mechanisms. She has participated in many research projects and is in charge the project of the China Postdoctoral Science Foundation. She has published more than 10 publications on international journals and conferences during the past 5 years. 